\title{
Subliminal Paradigmatic on Designing Character in Games
}

\author{
Sulaiman Hakim Lubis, \\ Animation Program, Visual Communication Design \\ Department, School of Design, Bina Nusantara University, \\ Jakarta, Indonesia 11480 \\ Sulaiman.Lubis@binus.edu
}

\author{
Tubagus Zufri, \\ Animation Program, Visual Communication Design \\ Department, School of Design, Bina Nusantara University, \\ Jakarta, Indonesia 11480 \\ Tubagus.Zufri@binus.ac.id
}

\begin{abstract}
Not only rely on aesthetics alone, but character design also requires things that underlie decision making in the design process. The consideration may refer to the type of story, style and appearance that also refers to the form, which is part of the elements of design, but other than those designers must also be paid attention to other things that related with the problem that occurs when the game is played. Games will be played by players who in fact have experiences that will later be brought into the game and instead bring experience from within the game to the real world. This unconscious condition can also be used as a design consideration.
\end{abstract}

Keywords- Character Design, Game, Design Elements, Experience, Unconscious

\section{INTRODUCTION}

If we look now, game players who play games in today's game world are like an iceberg, it looks small in number but in fact many in number and have a classification of interests towards different games. This means that someone's interest in the game will be very difficult to measure because now the game is global so it takes a great effort to be able to classify players based on the game they play.

The author sees many things in game design that can be discussed through the semiotic viewpoint. As stated by Neiva and Romano [1] "Signs, cues, and symbols work together in the game and are created to immerse the player in a world that must be constantly reinterpreted as the hinting of signs of something else." Many things such as signs, symbols and signs can be used as a reference for game players to continue to play that game. The game is played by players from a character perspective provided by the game and selected by players that makes players will experience the journey according to their game play. A character will experience the journey throughout the game and tied to the game mechanics that surround the game itself. Decision-making is the part that will always appear in the game and all options taken will be related to the risks that accompany it. For example in a game shooter during an attack (eg. a zombie entourage) the player is faced with the option of reloading when the bullet is still 5 or shooting the bullet effectively and then reloading. Both have different consequences, assuming that the first option will be at risk of running out of ammunition, while the second option will risk the loss of life. In an RPG game, for example, those choices are a logical set of options. a) Subliminal Paradigmatic

The plot of the story in a game requires a carrier that allows game design to be accepted by the player and makes it endure to play.

"The character design is not the only "key visual" in the game. Character in Game is an ideological representation of the game makers are designed in a way to be able to communicate with the audience. [2].

The carrier that allows communication between the game and the player is actually an information transaction process that was originally present as information about the existence of character, profile, environment, mission, navigation, strategy, level, enemy and the entire universe of the game.

In a game that requires players to choose the role they play until the game ends, players unconsciously merge themselves into the role provided by the game. Since the player chooses toplay the game.

b) Visual Paradigmatic

At the beginning when a player gets information about a new game, he will get a glimpse into the genre, plot of the story, who is the main character in the game. Furthermore, the player will be bombarded by visual information about the game and may determine the decision to play it or not.

"To some, winning or losing a game occupies an important social function, as it is a source of personal pride." [3]

The design of the characters will have much effect on the fun that comes when playing the game. Designing with rigorous and in accordance with the stages of good designing will make the players lose consciousness on reality and will take it to the subliminal phase.

"Powerful players-characters are often those that speak to many players'real-life hopes, fears, and issues. They offer players a chance to enact them and explore possibilities. "[4]

Character Design in Psychological Experience Perspective In a game, the role of the player and the character that is played has a very strong bond and even make a combination of both as a determinant of the continuity of the game universe. Deeper characters in the game are the vortex 
centers of experience offered in game play. The in- game character is an intermediary so players can feel what's going on in the game world. To be able to produce a good character is needed analysis of the reaction of players psychologically to the game characters played.

Game has its own world and reality but the games that are produced these days often have stories that are still a reflection of human life in their daily lives. As a human beings in general individuals who are in the game (game characters) are always at the intersection and faced with the decision making which will then be the experience in making choices and making the next decision.

Here are four layers of psychological experience experienced by game players associated with the game characters they play.[4]

1) Visceral Feedback, an experience that appears as it is in the game played when the player plays the character he chooses.

2) Cognitive Immersion is a model of self-adjustment between players with characters played so that players can adjust the problem-solving strategy encountered with the ability possessed by the game characters it plays.

3) Social Affordances, the condition of the existence of characters in the game world. Suppose the character in the game world is a former police officer who sought the murderof the child and his wife.

4) Fantasy Affordances, the character is able to offer a fantasy experience that represents the hopes, fears and problems that may be present in the player's real life.

\section{RESEARCH METHODS}

This research uses qualitative method by doing observation as a way to deeply understand the problem and pair it with literature data as anchor. The analysis is done descriptively to the design that become the object of study.

The main focus of this paper is the character design from the point of view of the psychological relationship between the player and the character being played. By understanding that point, it is hoped that the understanding will be the starting point to produce game characters and assets that have the most unintelligible foundation for deeper excavation of design in game development.

\section{DISCUSSION}

The bonding conditions formed between the player and the characters it plays can occur in the first and second stages. Because at these stages the player enters the body of characters in the game which will subsequently become / transform into an individual who travels in the game world bound by applicable laws in the game world

At these stages of psychological experience, the role of character design is highly influential or, in other words, understanding of these stages will guide us as designers to make design decisions that will later be accepted by game players.

In the first stage the thing that happens is the presence of the character with all its attributes, clothing, special abilities / skills, weapons, gender, face, body. Furthermore, cultural backgrounds can also make a substantial contribution either from a recognizable culture (original culture adapted) or cultures designed specifically for the game.

"Clothes, weapons and abilities resembled characters' roles and the participants could easily identify themselves withcharacters." [5]

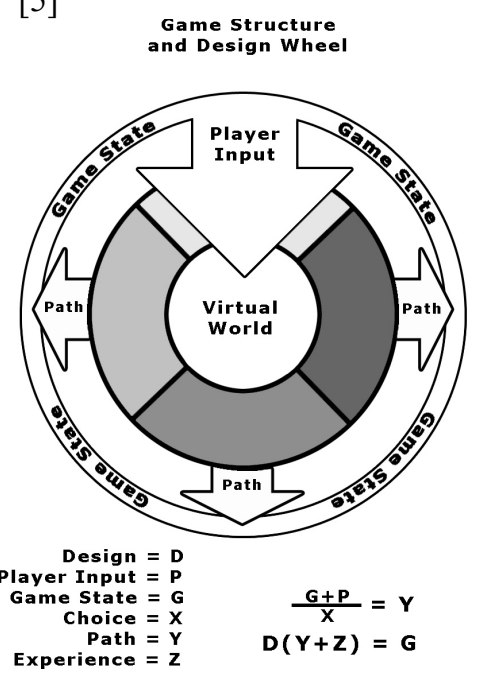

Fig. 1 Game and the Structure Design Wheel [1]

Character design can be referred to as a part that is in the realm of game $(G)$ will give input $(P)$ that leads on the path (Y). Input given to the virtual world is no longer a reality based existence owned by the player but is a form of input from fused awareness of the player will the existence/existence of the characters he played that are bound to the laws applied in the game is reflected from the gameplay.

Decisions concerning the design should be applied thoroughly and attached to characters designed through alternative sketches so as to respond to the demands presented earlier.

Many things can be done and each designer has his own way in embodying the concept that was originally verbal to be visual. But the most important thing that needs to be emphasized is that the design should be able to bring up new realities possessed by the game so as to generate a sense of trust in the player of the existence and ability of the character he played so that the purpose of playing the game can be achieved.

"The winner is not a strategist only, but the player who canpersonally do what the opponent cannot do. " [1] 


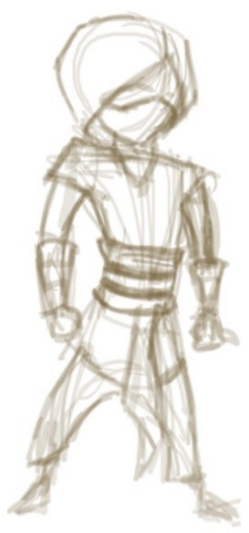

Fig. 2 Design Character sketch

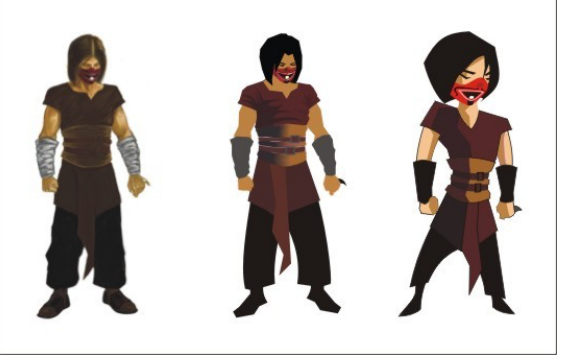

Fig. 3 Design Character Style Alternatives

When the designer in the early stages of design has no vision or purpose to answer the question of what psychological experience will be experienced by his players related to the character he will play, then the character as in the picture number 2 has no meaning other than visual entities. Here are four important points that can be used after the character designer in designing the character.

1) In the first stage. Which should be a consideration is, how to move with the physical condition that belongs to the game character, e.g. if the game character is a lizard. Is it quite exciting when the player moves and sticks to the wall? What abilities or strengths do you have? Is it equipped with weapons? How many weapons and how to get them? What kind of movement do you have? Etc. The more questions that can be answered, then the character will feel more real so that players will be able to integrate into the character.

2) In the second stage, when the phase of physical bonding has occurred, the question that arises in designing a character is: Does the player's way of thinking can also unite with the way game characters think? Will he then be able to feel that the character he is playing is united with his mind? Is the player's strategy in solving problems or obstacles to match the mechanics on gameplay?.

3) Then in the third stage, the question that should arise is: Does the player feel part of the social order in the gaming world he enters, how do players connect with the environment and also with all the NPCs in the game he is playing?

4) So in the fourth stage that should be a concern is: Whether players will be interested to live the life of the game character with all the provisions that have been outlined in the gameplay.

In the process of designing game characters in general and their relation to character design, there are several important characters besides the main character. One of these characters is what we know as nonplayer characters (NPC's) as mentioned earlier in point three. NPC's are all characters other than the characters played by players. These characters have their respective roles can be said to be a social role in building in-game reality. Therefore, each NPCs must be well designed. With the level of design with the same seriousness as the main character design but with a different psychological approach according to the role they play.

In designing these characters the social role of the character occurs when there are a series of behaviors and attitudes that emerge reciprocally with the conditions of taking into account each other's position. This can help to present scenes / situations that are social relations, such as interdependence / hand in hand in dealing with obstacles, power dynamics between social groups based on their hierarchy and conditions that unite one individual with another. At this stage awareness of social roles is very important because with this awareness conflict and misunderstanding can be avoided. In a game, conflicts that are not designed and misunderstandings that are outside the concept are a failure. The behavior of the characters played on the NPCs reflects the existence of the player that must be realized in a form that is integrated with the game world that is played, because only then will the player get experience from the game he plays.

Comprehensive understanding of the social role of the characters will greatly help visual design which of course has a visual hierarchy in presenting the visualization of characters both player characters and NPCs to reinforce the mission that must be completed and the ability inherent in each character. The relationship that occurs between the Player character and NPCs is very dependent on the initial concept of a game made, but the role of visual design will be able to bridge the story or experience arranged in the storyline.

"The story should be treated well, presenting the conflict and climax as well as visual support to the concept" [2] 


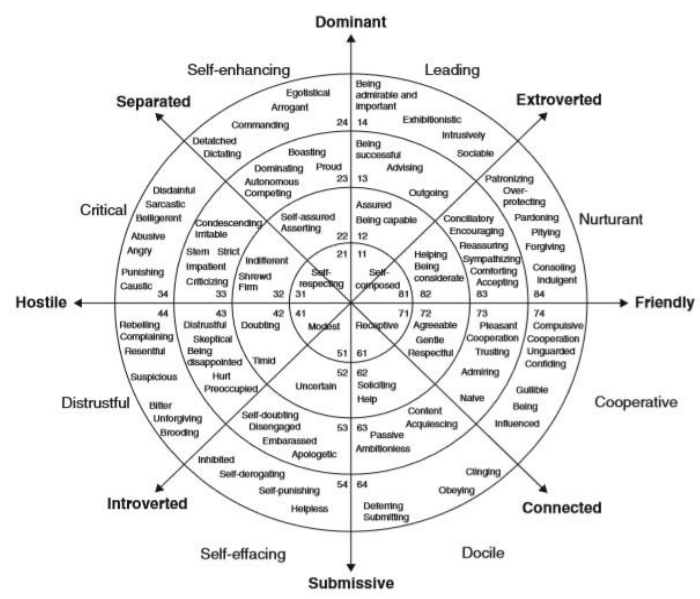

Figure 3 : The Interpersonal Circumplex [6]

"Your characters won't be alone, of course - they are going to interact with each other" $[6]$

The process of designing a character that can produce a strong bond with the player, should consider the psychological aspects that refer to the target market. This is very important because gaming is not enough to rely solely on the dynamic, mechanical and aesthetic aspects felt by the developer team, which may not be the market share of the created game. They must understand how to build bridges for mechanical, dynamic and aesthetic designed to touch the psychological side of the candidate.
Good character design will bring players to feel whatever is supposed to happen in the virtual world. If it does not happen then things that must be evaluated or traced back is the stages of psychological experience that occurred could be confusing.

Understanding of the social role of each character that exists allows the presence of the game as a world that resembles the concept of reality that is actually not real and only an idea of existence with mere artificial arrangements that make the player experience the movement of the soul and transform into a person who plays a role in a condition artificially where he can artificially control the situation as he might not be able todo in the real world.

\section{REVERENCE}

[1]. Neiva, E., \& Romano, C. (2007). The Semiotic Immersion of Video Games, Gaming Technology and Interactive Strategies. The Public Journal of Semiotics, 31-49.

[2]. Hilman, D., Zufri, T., \& Pratama, W. (2016). Character Design as Bridging Tools of Ideological Message in Game. 1st International Conference on Game, Game Art and Gamification (ICGGAG), 1-4.

[3]. Malliet, S. (2007). The Challenge of Video Game to Media Effect Theory. Leuven, Belgie: K.U.Leuven

[4]. Isbister, K. (2006). Better Game Characters by Design : A Psychological Approach. San Fancisco: Morgan Kaufmann Publishers.

[5]. Gast, A. (2017). Identification with Game Characters :Effects of visual attributes on the identification. Stockholm: Södertörns högskola.

[6]. Schell, J. (2008). The Art of Game Design. Burlington, MA 01830, USA: Morgan Kaufman Publishers. 